\title{
Kinematic and Dynamic Analysis of a 2-PRR Parallel Mechanism on Posture Adjustment for Aero-engine Installation
}

\author{
Zhe Zhao ${ }^{1, a^{*}}$, Zhendong Liu ${ }^{1, b}$ and Hang Gao ${ }^{2, c}$ \\ ${ }^{1}$ AVIC Shenyang Engine Design and Research Institute, Shenyang 110000, China \\ ${ }^{2}$ Dalian University of Technology, Dalian 116024, China \\ asky_03152006@126.com, ’dongz_1122@163.com, 'cgaohang@dlut.edu.cn
}

Keywords: Aero-engine; Parallel mechanism; Kinematics analysis; Dynamics analysis; Automatic installation

\begin{abstract}
Facing the precise fast posture adjustment problems of aero-engine for their large dimension, heavy weight, low stiffness, small installation space, as well as high precision and high efficiency of assembly task requirements, a new 2-PRR planar parallel mechanism is proposed to meet the elevation and inclination work in the automatic installation process for aero-engine, which is carried out by kinematics analysis to prove its feasibility. Applying multi-rigid system dynamics theory, its dynamic model is established and analyzed under working conditions of high inertia, high eccentric load to do a comparative analysis on the force process trends of each component during four work postures: original position, left inclination, right inclination, vertical elevation. According to the analysis results, conduct structural design and establish the multi-degree elevation and inclination posture adjustment platform, which reaches the high motion precision level of posture adjustment in the experimental accuracy test with eccentric load and error optimized compensation to provide a significant foundation on further development of a new multi-degree, automatic precise equipment for aero-engine installation.
\end{abstract}

\section{Introduction}

At present, the major aero-engine installation task is performed by a number of workers under manual collaboration with a simple non-powered mechanical vehicle, whose low productivity cannot meet the growing demand for mass production. The aero-engine belongs to huge components with large dimension, heavy weight, low stiffness, narrow installation space, as well as external complex structure forbidding any clash and collision, which brings up the great difficulty and challenge in the high precision and high efficiency of manufacture and maintenance. So the development for automatic posture adjustment platform of aero-engine installation has become one of the problems on installation capacity and technique to be solved and improved.

Compared to serial mechanism with large space, complex structure, low stiffness, low carrying capacity, parallel mechanism with few degrees of freedom (DOF) possesses the advantages of simple structure, high precision, high load capacity, favorable dynamic response, simple location inverse solution [1-3], which is more suitable for large mechanical movement and posture adjustment operation. For this reason, in recent years many scholars have conducted in-depth study of the theory and application in this regard [4-11]. And among few DOF parallel mechanisms, 2-PRR (P means movement deputy, $\mathrm{R}$ means rotation deputy) planar parallel mechanism has been widely used in the field of parallel machine tools, robots and assembly table with high-speed, high-precision, etc [12-14]. Therefore, a new 2-PRR planar parallel mechanism for automatic aero-engine installation is proposed to develop the multi-degree elevation and inclination posture adjustment platform.

Fig. 1 shows the multi-degree elevation and inclination posture adjustment platform consisting of three parts from top to bottom: translation mechanism, rotation mechanism, elevation and inclination mechanism (2-PRR planar parallel mechanism), which has four posture adjustment functions respectively: Y-axis horizontal movement, Z-axis vertical rotation, Z-axis vertical elevation and B-axis 
around inclination. The platform installed on automatic equipment can be operated on multi-axis CNC system to meet various movement needs of posture adjustment, which is convenient and efficient in the installation of aero-engine.

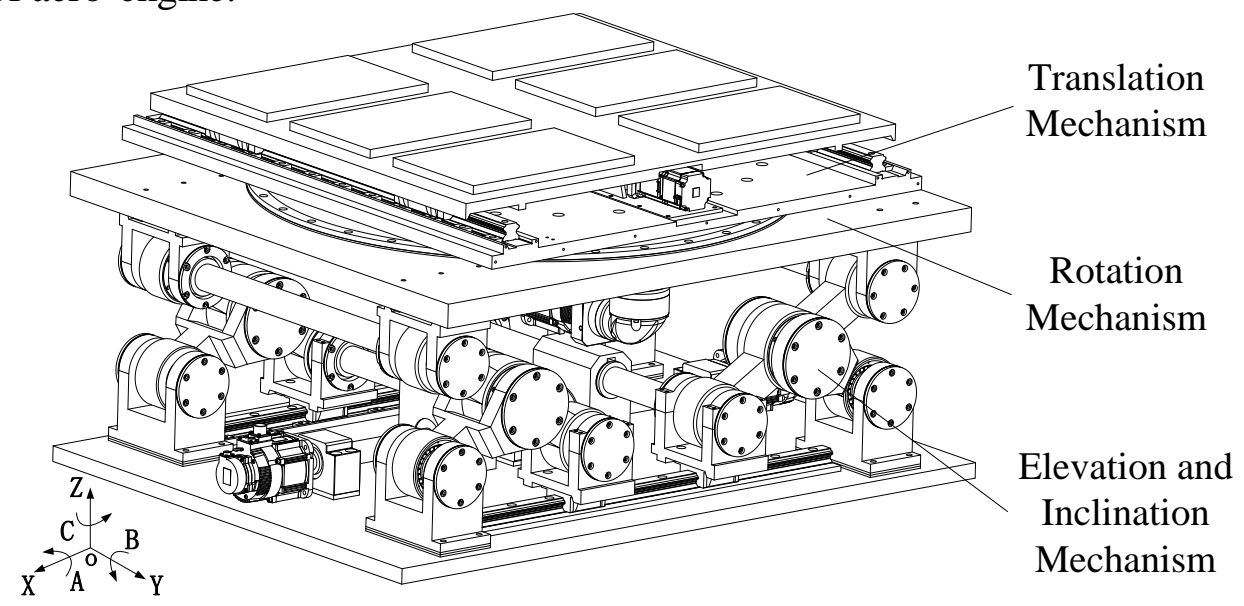

Fig.1 Multi-degree elevation and inclination posture adjustment platform

In order to optimize the dynamic characteristics of the platform, this paper applies multi-rigid system dynamics theory and Adams software to establish the dynamic model and analyze comparatively the force characteristics of each platform component under working conditions of high inertia, high eccentric load with four work posture processes: original position, left inclination, right inclination, vertical elevation. Then structural design is carried out based on the analysis results and multi-degree elevation and inclination posture adjustment platform is built up, which is tested in the load accuracy measurement experiment and the actual movement deviation is optimized by error compensation to obtain quite high movement accuracy. The results will lay the significant foundation on the development of new multi-degree, automatic precise equipment for aero-engine installation and play as an important guiding role.

\section{Kinematics analysis of 2-PRR planar parallel mechanism}

As is shown in Fig.2, it is the new 2-PRR planar parallel mechanism presented in this paper, which is composed of a moving table, a fixed table, left and right chains which are both the crank and slider mechanisms connected with the fixed table respectively through movement deputy $\mathrm{A}_{1}\left(\mathrm{~A}_{2}\right)$ and rotation deputy $\mathrm{C}_{1}\left(\mathrm{C}_{2}\right)$. The top of left chain is connected with fixed table through rotation deputy $\mathrm{B}_{1}$ while the top of right chain is connected with moving table through movement deputy $\mathrm{B}_{2}$ to eliminate the over-constrained problem. Therefore, this mechanism is called 2-PRR parallel mechanism whose movement deputy $P\left(A_{1} 、 A_{2}\right)$ is the driver deputy.

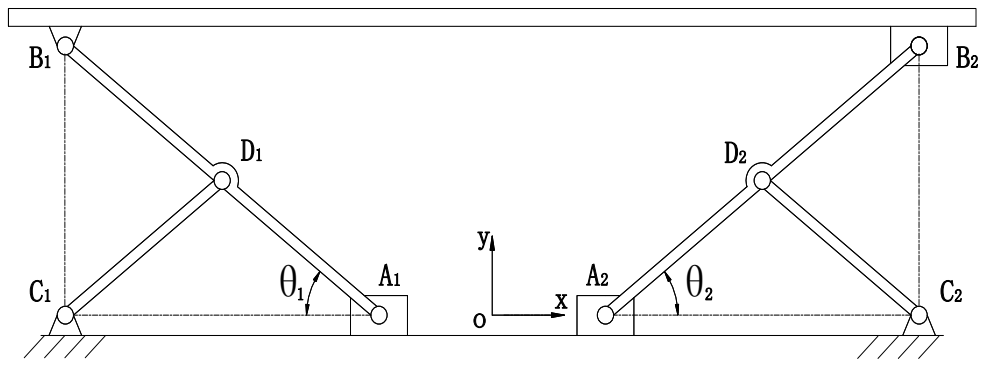

Fig.2 2-PRR planar parallel mechanism

To verify the posture adjustment movement feasibility of 2-PRR parallel mechanism designed, the kinematics model is established and the analysis is carried out. The freedom solution of 2-PRR parallel mechanism is as follows:

$$
F=(6-q) n-\sum_{k=q+1}^{5}(k-q) p_{k}=2(\mathrm{q}=3, \mathrm{n}=3, \mathrm{k}=5)
$$

Formula: $\mathrm{n}-$ number of active members 

$\mathrm{q}-$ number of public restraints
$\mathrm{k}-$ level of motion deputies
$\mathrm{p}$ —number of deputy constraint conditions

The 2-RRR parallel mechanism takes two servo motors as the prime mover of the motion whose freedom is equal to that of the chains, which proves that the 2-RRR parallel mechanism can meet the design conditions and posture adjustment movement is feasible.

\subsection{Inverse solution of kinematics}

Fig. 2 selects the center point $\mathrm{O}$ on the fixed table of the parallel mechanism as the original point to establish the rectangular coordinate system $\{\mathrm{O}-\mathrm{XY}\}$, where $\mathrm{A}_{\mathrm{i}} \mathrm{B}_{\mathrm{i}}=2 \mathrm{r}, \mathrm{C}_{\mathrm{i}} \mathrm{D}_{\mathrm{i}}=\mathrm{r}, \mathrm{OC}_{\mathrm{i}}=\mathrm{c}$, and the angle between $A_{i} B_{i}$ and $A_{i} C_{i}$ is $\theta_{i}$. The length of both left and right crank are half that of the link, which means $\mathrm{C}_{\mathrm{i}} \mathrm{D}_{\mathrm{i}}=1 / 2 \mathrm{~A}_{\mathrm{i}} \mathrm{B}_{\mathrm{i}}(\mathrm{i}=1,2)$.

According to the judgment rule of right triangle, $\triangle A_{i} B_{i} C_{i}$ always remains the right one to make its center point $B_{i}$ keep elevating vertically. So the two movement deputies $P\left(A_{1}, A_{2}\right)$ are controlled by servo motors in order to achieve the two DOF motions of moving table in the mechanism plane: elevation and inclination.

The input vector of parallel mechanism $\boldsymbol{a}_{i}$ means the coordinate value of two slider center points $\mathrm{A}_{\mathrm{i}}$ of the fixed table in the X-axis direction, where $\boldsymbol{a}=\left[\mathrm{a}_{1} \mathrm{a}_{2}\right]^{\mathrm{T}}$, The output vector of parallel mechanism $\boldsymbol{b}_{\boldsymbol{i}}$ means the coordinate value of two joint center points $B_{i}$ of the moving table in the $\mathrm{Y}$-axis direction, where $\boldsymbol{b}=\left[b_{1} b_{2}\right]^{\mathrm{T}}$.

According to the geometric constraints of $\triangle A_{i} B_{i} C_{i}$, we can get that $b_{i}=\sin a_{i} A_{i} B_{i}$ and $\cos a_{i}=A_{i} C_{i} / A_{i} B_{i}$. The kinematics inverse equation of 2-RRR parallel mechanism by calculation conversion is as follows:

$$
\left\{\begin{array}{l}
a_{1}= \pm \sqrt{4 r^{2}-b_{1}{ }^{2}}-c \\
a_{2}= \pm \sqrt{4 r^{2}-b_{2}{ }^{2}}+c
\end{array}\right.
$$

There exist four groups of inverse solutions in equation (1) totally shown in Fig.3:

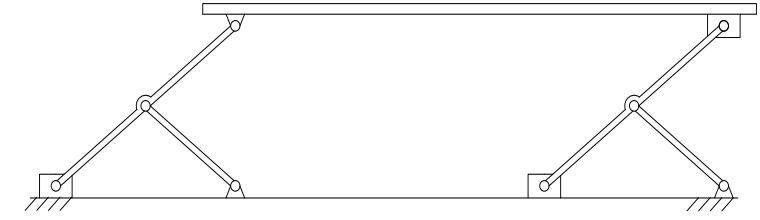

(a)

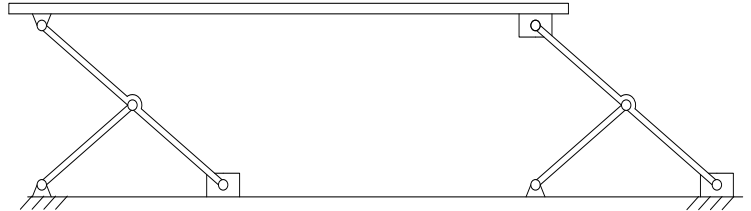

(c)

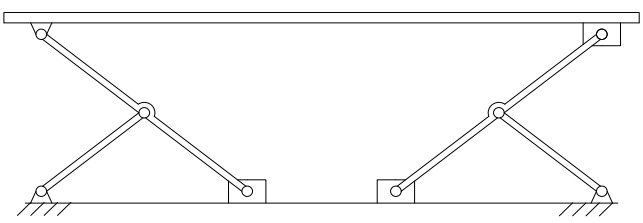

(b)

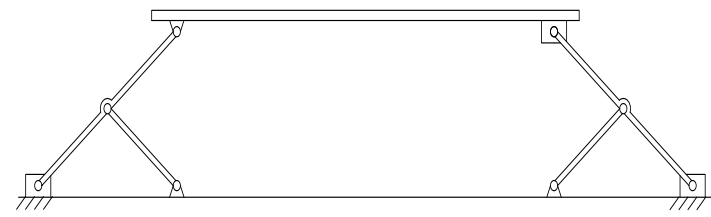

(d)

Fig.3 Kinematic inverse solution of 2-PRR planar parallel mechanism sketch

Comparing four groups of institutions obtained by inverse solution, the mechanism (a) and (c) of moving table cannot be considered for lacking of enough stability. Moreover, taking into account the space limitation and the features of the mechanism (b) and (d), it is found that the mechanism (b) can attain larger working space on smaller fixed table to proves the feasibility of this designation further. The sign " + " is taken in the first equation of formula (1) when the sign "-" is taken in the second equation. Thus the kinematic inverse solution of 2-PRR planar parallel mechanism is finally acquired as follows:

$$
\left\{\begin{array}{l}
a_{1}=\sqrt{4 r^{2}-b_{1}^{2}}-c \\
a_{2}=-\sqrt{4 r^{2}-b_{2}^{2}}+c
\end{array}\right.
$$

\subsection{Positive solution of kinematics}


Known the input vector $\boldsymbol{a}_{\boldsymbol{i}}$, the output vector $\boldsymbol{b}_{\boldsymbol{i}}$ of $\triangle \mathrm{A}_{\mathrm{i}} \mathrm{B}_{\mathrm{i}} \mathrm{C}_{\mathrm{i}}$ in the 2-PRR parallel mechanism can be calculated to obtain:

$$
\left\{\begin{array}{l}
b_{1}=\tan a_{1}\left(c+a_{1}\right) \\
b_{2}=\tan a_{2}\left(c-a_{2}\right)
\end{array}\right.
$$

The positive kinematics solution of the parallel mechanism can be got by calculating conversion into formula (3):

$$
\left\{\begin{array}{l}
b_{1}=\sqrt{4 r^{2}-\left(c+a_{1}\right)^{2}} \\
b_{2}=\sqrt{4 r^{2}-\left(c-a_{2}\right)^{2}}
\end{array}\right.
$$

The inclination angle between moving table and fixed table is as follows:

$$
\theta=\arctan \left(\frac{\sqrt{4 r^{2}-\left(c-a_{2}\right)^{2}}-\sqrt{4 r^{2}-\left(c+a_{1}\right)^{2}}}{2 c}\right)
$$

Taking use of computer programming language with the positive and inverse kinematics solution of the elevation and inclination platform by calculating analysis, the theoretical algorithm of subsequent digital posture adjustment table can be provided to achieve automatic and precise control of installation trajectory for aero-engine.

\section{Dynamics modeling of 2-PRR elevation and inclination mechanism}

\subsection{Virtual prototype of platform}

Applying 2-PRR planar parallel principle to establish the platform of 2-PRR elevation and inclination mechanism, its principle sketch of virtual prototype is shown in Fig.4. The platform consists of moving table, fixed table, crank, link, slider and joint, which is connected with two 2-PRR parallel mechanisms symmetrically to form the closed frame platform with high strength and high stability.

The virtual prototype of platform is composed of 47components and added up 55 contact constraints including 6 transitional deputies, 16 rotating deputies and 33 fixed deputies. According to actual working conditions, the two strengthened shafts connected to sliders are fixedly set as the driver bar and movement deputy is added up which can be controlled by simulation motor. So the virtual prototype will possess the determined motion law and can be conducted by dynamic characteristic analysis.

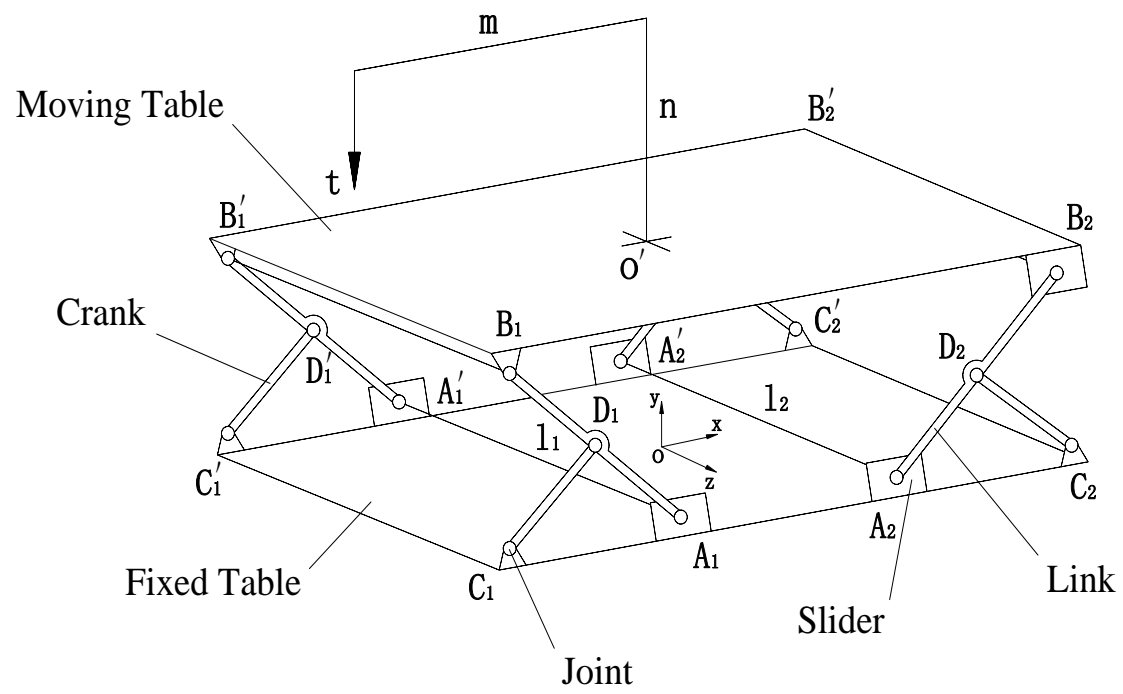

Fig.4 Principle diagram of 2-PRR elevation and inclination mechanism

Considering the actual work needs, the platform should be able to meet the high inertia load and eccentric posture adjustment. To facilitate the analysis and comparison, set the initial conditions as: eccentric load $\mathrm{t}=2000(\mathrm{~kg}), \mathrm{m}=600(\mathrm{~mm}), \mathrm{n}=800(\mathrm{~mm})$; the material of each components is $45 \#$ and link $A_{i} B_{i}=480(\mathrm{~mm})(i=1,2)$, crank $C_{i} D_{i}=240(\mathrm{~mm})$, driver link $1_{1}=l_{2}=900(\mathrm{~mm})$, the distance between 
the joint centers of the moving and fixed table $B_{1} B_{2}=C_{1} C_{2}=1200(\mathrm{~mm})$. Take use of virtual prototype to conduct simulation analysis as follows.

\subsection{Dynamics model of mechanism}

Apply the Lagrange equation method of multi-rigid system dynamics theory in the Adams software, the dynamic equation of the posture adjustment platform is established and select the three rectangular coordinates of each center of mass in the inertial reference system of the platform, and take Cartesian coordinates of each center of mass position and Euler angles reflecting rigid orientation as generalized coordinates: $\mathrm{q}_{\mathrm{i}}=[\mathrm{x}, \mathrm{y}, \mathrm{z}, \psi, \theta, \varphi], \mathrm{q}=\left[\mathrm{q}_{1}{ }^{\mathrm{T}}, \mathrm{q}_{2}{ }^{\mathrm{T}}, \ldots, \mathrm{q}_{\mathrm{n}}{ }^{\mathrm{T}}\right]^{\mathrm{T}}$. By means of Lagrange multiplier method to establish the system dynamic equations as follows:

$$
\frac{d}{d t}\left(\frac{\partial T}{\partial \dot{q}}\right)^{T}-\left(\frac{\partial T}{\partial q}\right)^{T}+\varphi_{q}^{T} \rho+\theta_{\dot{q}}{ }^{T} \mu=Q
$$

The complete constraint equation:

$$
\varphi(q, t)=0
$$

The incomplete constraint equation:

$$
\theta(q, \dot{q}, t)=0
$$

Formula: $\mathrm{T} —$ - kinetic energy of system

Q__ generalized force array

q_- generalized coordinate array of system

$\rho$ - Lagrange multiplier array of complete constraint

$\mu-$ Lagrange multiplier array of incomplete constraint

\subsection{Motion simulation of working conditions}

Set the test time is $60(\mathrm{~s})$ and the sampling frequency is $0.1(\mathrm{~Hz})$. The step function is applied to further conform to the simulation process in real working conditions by means of constituting cubic polynomials. The working postures of platform consist of four processes: original position, left inclination, right inclination, vertical elevation. The four different postures can be determined by the following driver function.

Add up the driver function to the movement deputy of left elevation and inclination mechanism: step(time, $0,0,5,-80)+$ step (time, $5,0,15,160)+$ step(time, 15,0,20,-80)+step(time, 40,0,45,-80)+step (time, 45,0,55,160)+step(time,55,0,60,-80);

Add up the driver function to the movement deputy of right elevation and inclination mechanism: step(time, 20,0,25,80)+step(time, 25,0,35,-160)+step(time, 35,0,40,80)+step(time, 40, 0, 45, 80)+st ep(time,45,0,55,-160)+step(time,55,0,60,80);

The driver function means the elevation and inclination mechanism stays in the initial position, inclines left in 0-20 (s), then inclines right in 20-40 (s), elevates in 40-60 (s) and at last returns to the original place to finish the four complete posture movements in order: original position, left inclination, right inclination, vertical elevation.

\section{Force process analysis of each joint for platform}

Analyzing the joint force processes of the elevation and inclination mechanism in the four working postures: original position, left inclination, right inclination, vertical elevation, it is found that due to the particularity of loading, the force of each joint in the $\mathrm{Z}$ axis direction nearly reaches zero which can be neglected, and the radial force just need be analyzed. Simulating the four postures of the elevation and inclination mechanism, the force process data of each joint in the $\mathrm{X}$ and $\mathrm{Y}$ axis direction can be acquired and the maximum force of each joint is shown in the following Tab.1. 
Tab.1 The maximum joint force of elevation and inclination mechanism (Unit: $\mathrm{N}$ )

\begin{tabular}{cccccccccc}
\hline $\begin{array}{l}\text { Working } \\
\text { Posture }\end{array}$ & \multirow{2}{*}{ Direction } & $\mathrm{A}_{1}$ & $\mathrm{~A}_{2}$ & $\mathrm{~B}_{1}$ & $\mathrm{~B}_{2}$ & $\mathrm{C}_{1}$ & $\mathrm{C}_{2}$ & $\mathrm{D}_{1}$ & $\mathrm{D}_{2}$ \\
\hline Original & $\mathrm{X}$ & -14580.1 & 2052.3 & 0 & 0 & 14580.1 & -2052.3 & 14580.1 & -2052.3 \\
Position & $\mathrm{Y}$ & 264.3 & 264.3 & -11146.4 & -1341.8 & 11520.5 & 1715.9 & 11307.6 & 1439.6 \\
Left & $\mathrm{X}$ & -14754.7 & 5634.2 & 157.6 & -156.5 & 14818.0 & -5477.4 & 14818.0 & -5477.5 \\
Inclination & $\mathrm{Y}$ & 387.7 & 314.1 & -11283.0 & -1505.2 & 11706.7 & 1831.1 & 11493.8 & 1554.3 \\
Right & $\mathrm{X}$ & -37301.4 & 2180.5 & -102.9 & 100.3 & -2101.7 & -2101.7 & 37402.9 & -2101.7 \\
Inclination & $\mathrm{Y}$ & 364.0 & 326.0 & -11437.6 & -1442.2 & 1754.6 & 1754.6 & 11631.8 & 1478.3 \\
Vertical & $\mathrm{X}$ & -36395.7 & 5138.2 & 0 & 0 & 36395.4 & -5137.7 & 36395.5 & -5137.9 \\
Elevation & $\mathrm{Y}$ & 264.9 & 264.9 & -11154.4 & -1349.8 & 11529.5 & 1724.9 & 11316.3 & 1448.1 \\
\hline
\end{tabular}

Observing Tab.1, the force of joint $A_{i}, B_{i}, C_{i}, D_{i}$ of the four working postures is the maximum in the $\mathrm{Y}$ axis direction except the joint $\mathrm{B}_{\mathrm{i}}$, and each joint force of left elevation and inclination mechanism is larger than that of right elevation and inclination mechanism. Moreover, the joint force reaches the maximum in the $\mathrm{X}$ axis direction, so take left elevation and inclination mechanism as the main reference object to analyze. The maximum force of the joint $A_{1}$ of left elevation and inclination mechanism is $-36395.7(\mathrm{~N})$ in the $\mathrm{X}$ axis direction when elevating vertically for the platform; the maximum force of the joint $\mathrm{B}_{1}$ is $-11437.6(\mathrm{~N})$ in the $\mathrm{Y}$ axis direction when inclining right for the platform; the maximum force of the joint $\mathrm{C}_{1}$ is $36395.4(\mathrm{~N})$ in the $\mathrm{X}$ axis direction when elevating vertically for the platform; the maximum force of the joint $\mathrm{D}_{1}$ is $37402.9(\mathrm{~N})$ in the $\mathrm{X}$ axis direction when inclining right for the platform. The analysis results of each joint force of elevation and inclination mechanism can provide strong support for structure design and bearing characteristics optimization further.

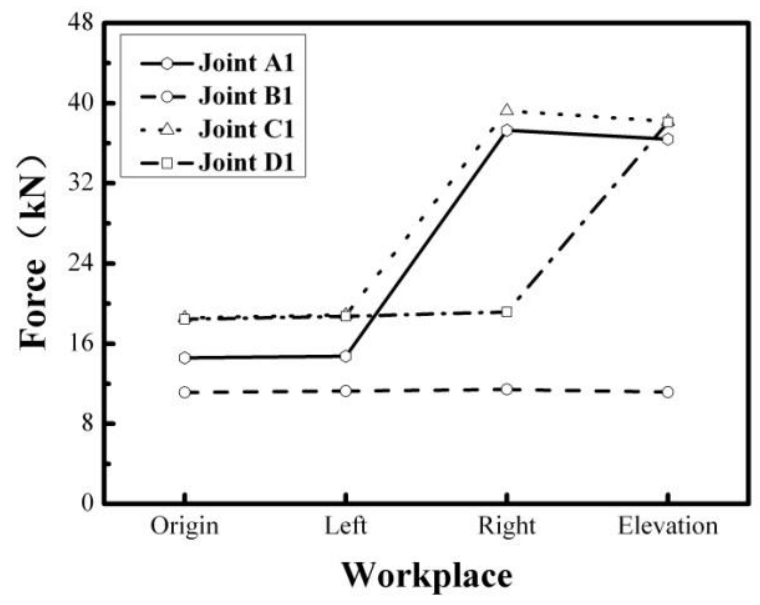

(a)Left elevation and inclination mechanism

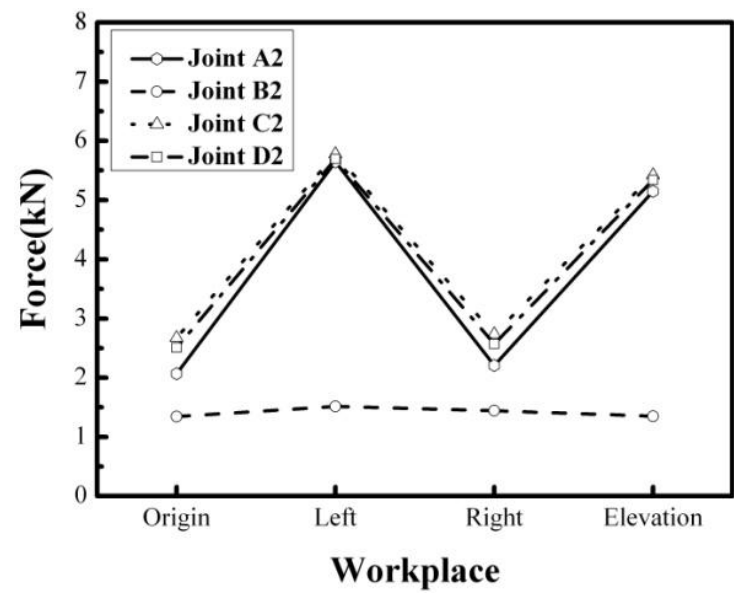

(b)Right elevation and inclination mechanism

Fig.5 Joint force curves of elevation and inclination mechanism

Comparing each joint resultant force (absolute value) of left elevation and inclination mechanism with that of right elevation and inclination mechanism in the four working conditions shown in Fig.5, the joint forces of left elevation and inclination mechanism are all larger than that of right elevation and inclination mechanism, and the forces of joint $A_{i} 、 C_{i} 、 D_{i}$ changes much larger than that of joint $B_{i}$, but the force of joint $B_{i}$ does not change too much.

Shown in Fig.5 (a), the joint $A_{1}$ force of left elevation and inclination mechanism changes almost the same as the joint $\mathrm{C}_{1}$, which changes greatly when the working posture inclines from left to right and the force increases significantly to the largest when inclining right, and then the force drops down when elevating vertically. The joint $\mathrm{D}_{1}$ force does not nearly make any changes, but comes to the largest when elevating vertically.

Shown in Fig. 5 (b), the forces of the joint $A_{2}, C_{2}, D_{2}$ change almost the same in the right elevation and inclination mechanism, which increase largely when the working posture moves from the original 
position to left inclination reaching the largest, and then decrease suddenly, soon afterwards increase greatly again to form the fluctuations. The four postures of elevation and inclination mechanism in the real working conditions is simulated by establishing the virtual prototype, the dynamic change parameters and characteristics of each joint force is analyzed to acquire when elevation and inclination mechanism is bearing the special working conditions of the high inertia and eccentric load, whose analyzing results will provide the important basis on achieving the precise and optimized control of mechanism.

\section{Accuracy measurement of loading experiment}
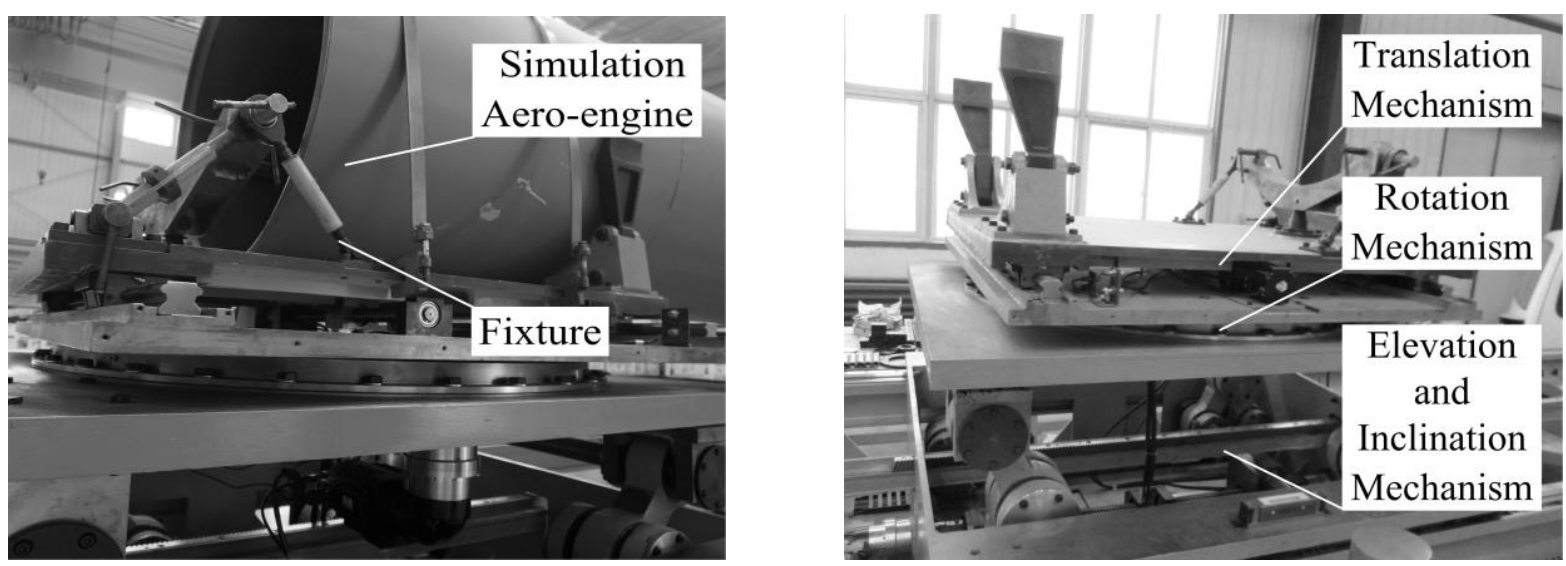

Fig.6 Simulation load test Fig.7 Elevation and inclination posture adjustment platform

Fig. 6 presents that the experiment platform is built up for application verification. The simulation aero-engine is set on the fixture of the multi-degree elevation and inclination posture adjustment platform, which is applied with load $\mathrm{t}=2000(\mathrm{~kg})$ and is fixed eccentrically based on the same load conditions as the motion simulation of Adams. Shown in Fig.7, the multi-degree elevation and inclination posture adjustment platform is established including translation mechanism, rotation mechanism, elevation and inclination mechanism and according to dynamic characteristic analysis results, the systematic structure design is carried out for the important joint, crank and link of the elevation and inclination mechanism. Also based on the inverse solution of kinematics, the computer language is applied in making programs to achieve automatic and numerical control.

And then the controlling measurement experiment of movement accuracy is carried out on the 2-PRR elevation and inclination mechanism, and the measurement content is the movement displacement accuracy of the slider input and the joint output, which is converted mathematically to the displacement deviation of joint output in the $\mathrm{Z}$ axis direction to make a comparison with theoretical calculation results.

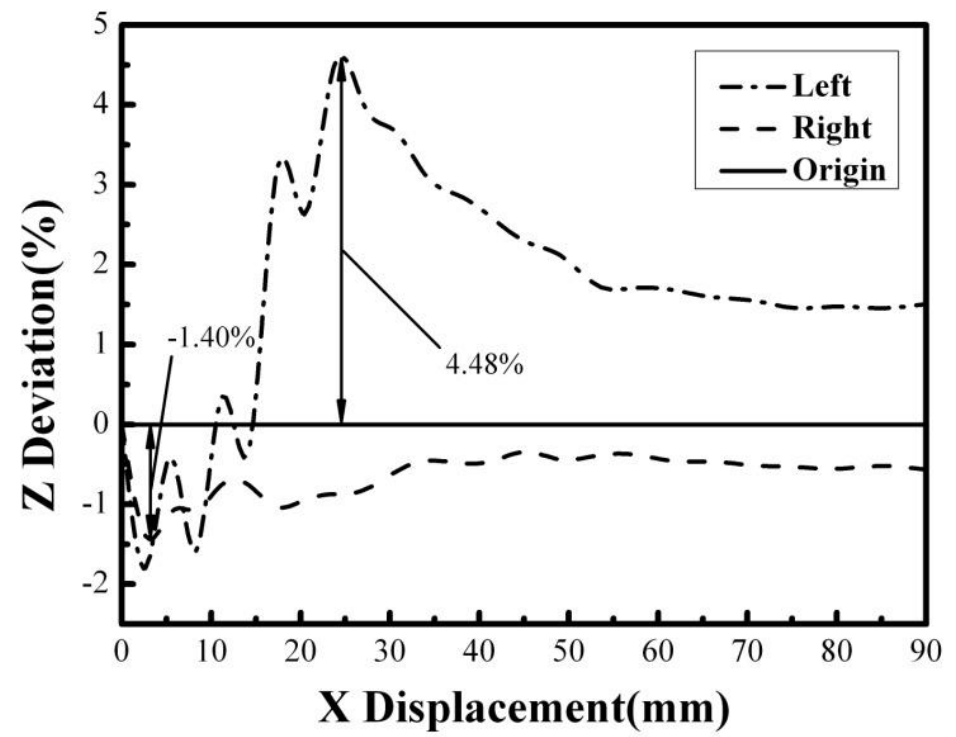


Fig. 8 Movement curve of elevation and inclination mechanism with load

Fig. 8 shows the actual and theoretical movement curves of left and right elevation and inclination mechanism, it is found that the movement deviation of left elevation and inclination mechanism is $4.48 \%$ which is larger when the movement deviation of right elevation and inclination mechanism is $-1.40 \%$. Then applying the inverse solution of kinematics, the actual movement deviation is optimized by error compensation to increase the posture adjusting accuracy further so that the movement deviation of the left and right elevation and inclination mechanism is controlled below $\pm 1 \%$. This illustrates that the elevation and inclination mechanism comes to the higher movement accuracy on the condition of high inertia and eccentric load and possesses a better posture adjusting effect, which verifies further the rationality and accessibility of structure design and automatic control and will hold a favorite applying prospect.

\section{Summary}

(1) a new 2-PRR planar parallel mechanism is proposed for precise installation of aero-engine, and based on this, the multi-degree elevation and inclination posture adjustment platform is developed with the four functions of radial movement, vertical rotation, vertical elevation, left and right inclination to meet the special conditions of high inertia and eccentric load;

(2) the kinematic analysis for 2-PRR planar parallel mechanism is carried out to obtain the positive and inverse solutions whose expression formulas are so simple to provide the theoretical algorithms for the numerical control of elevation and inclination mechanism;

(3) the software Adams is applied in carrying out the simulation dynamic analysis for elevation and inclination mechanism and the force change characteristics of each joint on the different working conditions are acquired to offer important references for structure design of the platform;

(4) the structure design is conducted for the platform by the dynamic analysis results and the multi-degree elevation and inclination posture adjustment platform is established, which is tested in the accuracy measurement of loading experiment and optimized by error compensation according to the kinematic analysis results to acquire high movement accuracy;

(5) the research results of this paper lay the technical foundation of developing multi-degree, intelligent, high-load, precise, and automatic equipment which will hold a favorite and broad applying prospect.

\section{References}

[1] QU Yun-xia, LI Wei-min, FAN Shun-cheng. Research overview of parallel mechanism configuration[J]. Machining Technology and Equipment, 2007, 5(2): 48-51.

[2] HAO Qi. Optimization Design and Dynamic Control of a 2-DoF Planar Parallel Manipulator[D]. Beijing: Tsinghua University, 2011.

[3] ZHANG Yue-min, XIE Gang. The study of parallel robot manipulator[J]. Industrial Robotics, 2008, 8(3): 8-9.

[4] HAN Shu-kui, FANG Yue-fa, GUO Sheng. Act kinematics performance analysis for lower degrees of freedom parallel manipulators[J]. Journal of mechanical Engineering, 2009, 45(9): 58-63.

[5] HU Ming. Kinematics models and development of control system of 2-PRR parallel machine[J]. Mechanical Engineering and Technology, 2012, 125: 367-372.

[6] R.Vilanova. Reference controller design in 2-DOF control[J]. Electrical Engineering, 2008, 90(4): 275-281. 
[7] T.Itul, D.Pisla, A.Stoica. Kinematics and design of a simple 2-DOF parallel mechanism used for orientation[J]. New Trends in Mechanism Science, 2010, 5: 39-46.

[8] WANG Su, HUAI Qi-wu, MIAO Xin-gang. Design and the volumetric error models of 2-DOF parallel manipulator[J]. Design and Research, 2012, 39(8): 7-11.

[9] PENG Bin-bin, LI Zeng-ming, Wu Kai. Spatial static stiffness analysis of 2-DOF translational parallel robots[J]. Journal of Nanjing University of Science and Technology, 2012, 36(3): $522-528$.

[10] WU Jun, Wang Jin-song, Li Tie-min. Dynamic analysis of the 2-DOF planar parallel manipulator of a heavy duty hybrid machine tool[J]. The International Journal of Advanced Manufacturing Technology, 2007, 34: 413-420.

[11] Yun-Joo, Nam, Myeong-Kwan Park. Workspace optimization and kinematic performance evaluation of 2-DOF parallel mechanisms[J]. Journal of Mechanical Science and Technology, 2006, 20(10): 1616-1625.

[12] WANG Li-ping, WANG JIN-song. Research status and prospects of the new parallel machine[J]. Aeronautical Manufacturing Technology, 2004, 6: 26-30.

[13] YANG Bin-jiu, CAI Guang-qi, LUO Ji-man. The state of research on limited-DOF parallel robot[J]. Machine Tool \& Hydraulics, 2006, 5(8): 202-205.

[14] ZHANG Gang, LIU Pin-kuan, DING Han. Optimal kinematic design of a 2-DoF translational parallel manipulator with high speed and high precision[J]. Intelligent Robotics and Applications, 2011, 7101: 445-454. 